\title{
Shorter Diagnosis-to-Treatment Interval in Diffuse Large B-Cell Lymphoma is Associated With Inferior Overall Survival in a Large, Population-Based Registry
}

Danielle N. Blunt, BMBS ${ }^{1,2}$; Liam Smyth, MD, FRCPath ${ }^{1,3}$; Chenthila Nagamuthu, MPH4; Evgenia Gatov, MPH ${ }^{4}$; Ruth Croxford, MSc ${ }^{4}$; Lee Mozessohn, MD'; and Matthew C. Cheung, MD, MSc ${ }^{1,4,5}$

\section{ABSTRACT}

Background: Because of prolonged screening requirements, patient and time-dependent selection have been proposed as potential biases in clinical trials. The screening process may exclude patients with a need for emergent treatment (and a short period from diagnosis to treatment initiation [DTI]). We explored the impact of DTI on overall survival (OS) in a population-based cohort of patients with diffuse large B-cell lymphoma (DLBCL). Patients and Methods: Using population-based administrative databases in Ontario, Canada, we identified adults aged $\geq 18$ years with DLBCL treated with rituximab-based chemotherapy for curative intent between January 2005 and December 2015. Cox regression and multivariable analyses were presented to evaluate the impact of time from DTI on OS, controlling for relevant covariates. Results: We identified 9,441 patients with DLBCL in Ontario; median age was 66 years, $53.6 \%$ were male, median number of comorbidities (Johns Hopkins aggregated diagnosis groups) was 10 (interquartile range [IQR], 8-13), and median DTI was 37 days (IQR, 22-61). Between treatment initiation and study end, $43 \%$ of patients died (median OS, 1 year; IQR, 0.4-2.8 years). Shorter DTI was a significant predictor of mortality $(P<.001)$. Compared with the shortest DTI period of 0-18 days, those who commenced therapy at 19-29 days (hazard ratio [HR], 0.75; 95\% $\mathrm{Cl}, 0.68-0.84), 30-41$ days ( $\mathrm{HR}, 0.70 ; 95 \% \mathrm{Cl}, 0.63-0.78), 42-57$ days $(\mathrm{HR}, 0.52 ; 95 \% \mathrm{Cl}, 0.46-0.58)$, and $58-180$ days $(\mathrm{HR}, 0.52 ; 95 \% \mathrm{Cl}$, $0.47-0.58)$ had improved survival. Increasing age (HR, 1.03; $95 \% \mathrm{Cl}$, 1.03-1.04), male sex (HR, 1.23; 95\% Cl, 1.14-1.32), and increasing number of comorbidities (HR, 1.12; $95 \% \mathrm{Cl}, 1.11-1.13)$ were associated with inferior survival. Conclusions: Among patients with DLBCL, shorter DTI was associated with inferior OS. Therefore, DTI may represent a surrogate marker for aggressive biology. Clinical trials with lengthy screening periods are likely creating a timedependent patient selection bias.

J Natl Compr Canc Netw 2021;19(6):719-725 doi: $10.6004 /$ jnccn.2020.7654

${ }^{1}$ Odette Cancer Centre, Sunnybrook Health Sciences Centre, Toronto, Ontario, Canada; ${ }^{2}$ Department of Haematology, Royal Adelaide Hospital, Adelaide, South Australia, Australia; ${ }^{3}$ Department of Haematology, St. Vincent's University Hospital, Dublin, Ireland; and ${ }^{4}$ ICES, and ${ }^{5}$ Division of Hematology/Medical Oncology, Department of Medicine, University of Toronto, Toronto, Ontario, Canada.

\section{Background}

Despite advances in treatment of diffuse large B-cell lymphoma (DLBCL), approximately one-third of patients will experience relapse after primary therapy, with known risk factors largely limited to disease biology. When treated with standard of care R-CHOP (rituximab/ cyclophosphamide/doxorubicin/vincristine/prednisone), the activated B-cell (ABC) subtype of DLBCL harbors inferior outcomes compared with the germinal center B-cell (GCB) subtype. ${ }^{1}$ ABC DLBCL likely represents a unique disease biology, with overexpression and constitutive activation of nuclear factor kappa B (NF-кB) within the BCR signaling pathway. ${ }^{2}$

To overcome the aggressive biology of ABC DLBCL, recent studies have explored adding targeted agents that inhibit the BCR pathway to R-CHOP. Although earlyphase studies showed promising results, more recent phase III trials specific to ABC or non-GCB DLBCL have failed to demonstrate improvements in survival. ${ }^{3,4}$ Furthermore, survival rates in the control arms of these studies (standard R-CHOP therapy) exceeded expected estimates based on historical studies. ${ }^{5}$ It has been postulated that prolonged screening periods and selective nonenrollment of high-risk patients into trials may be contributing to a time-dependent selection bias, thus decreasing the power of trials to identify beneficial effects of novel agents. In DLBCL, the expected time to screen for trial enrollment must be balanced against the clinical urgency for treatment initiation. Central pathology review to determine cell of origin, as mandated in many trial designs, necessitates longer screening periods. Maurer et $\mathrm{al}^{6}$ reported that in a prospectively enrolled cohort of patients with newly diagnosed DLBCL predominantly treated within academic settings, those with $>15$ days from diagnosis to treatment initiation (DTI) had lower-risk characteristics and improved event-free survival (EFS) at 24 months from diagnosis compared with patients treated more urgently. Furthermore, a retrospective, observational study of the US National 
Cancer Database (NCDB) reported reduced overall survival (OS) in patients treated within 7 days compared with those treated $>30$ days from diagnosis in several subtypes of aggressive lymphomas, including DLBCL, mantle cell, Burkitt, and peripheral T-cell. ${ }^{7}$

Whether a shorter DTI represents a surrogate marker for aggressive biology and its impact on OS remain less certain, particularly in a state-funded healthcare system. We evaluated the impact of DTI on OS among a large population-based cohort of patients with DLBCL in the era of rituximab.

\section{Patients and Methods}

All patients aged $\geq 18$ years with a new diagnosis of DLBCL in Ontario, Canada, were identified through administrative databases available at ICES, an independent, not-for-profit research institute whose legal status under Ontario's health information privacy law allows it to collect and analyze healthcare and demographic data, without consent, for health system evaluation and improvement. The Ontario Cancer Registry contains information on approximately $95 \%$ of all provincial cancer diagnoses; information on disease stage was collected from 2007 onward. The New Drug Funding Program is a registry of all publicly funded higher-cost infusional cancer therapies, including rituximab. Patient demographics were determined using the Registered Persons Database. For healthcare utilization, we used the Ontario Health Insurance Plan (OHIP) database, containing diagnostic and fee codes for physician services, the Canadian Institute for Health Information Discharge Abstract Database (CIHI-DAD), which contains information pertaining to all inpatient hospital discharges in Ontario, and the National Ambulatory Care Reporting System (NACRS), which captures emergency department and community-based ambulatory care. Information on cause-of-death was obtained from the Ontario Office of the Registrar General's death dataset and was available for all deaths occurring up to December 2016. These datasets were linked using unique encoded identifiers and analyzed at ICES.

This population-based retrospective cohort study included all patients diagnosed between January 1, 2005, and December 31, 2015, receiving a rituximab-containing chemotherapy regimen as part of first-line therapy with curative intent. Index date was defined as date of first rituximab treatment. Patients with transformed follicular lymphoma (FL) were included, provided they had not been previously treated. Patients with primary central nervous system lymphoma and HIV-associated lymphoma were excluded. Patients were followed until death, occurrence of a new primary cancer, or March 31, 2017. Comorbidities were identified using diagnostic codes found in inpatient discharge records (CIHI-DAD), emergency department visits (NACRS), and OHIP claims dated within 2 years prior to index date. The Johns Hopkins ACG system version 10.0 (Johns Hopkins University) was used to group diagnoses into Aggregated Diagnosis Groups (ADGs).

DTI was defined as the time from diagnosis to initiation of rituximab-based therapy. The primary outcome was OS, defined as time from the index date to date of death, new primary cancer, or end of study period. Cox proportional hazards regression analyses were used to identify predictors of OS. DTI was categorized into quintiles to ensure equal sample size across groups. We adjusted for the following covariates: age, sex, distance from the patient's postal code to treatment facility, neighborhood income quintile, rural dwelling status, number of ADGs, cancer type (DLBCL vs transformed FL), and type of treatment facility (nonteaching vs teaching). To exclude patients with treatment delays possibly unrelated to lymphoma, the analysis was limited to patients commencing therapy within 180 days of diagnosis date and who had available data on distance to treatment facility. In a secondary analysis, DTI was modeled using restricted cubic splines. ${ }^{9}$ All covariates were retained in the model, except for distance to treatment facility, which was not a significant predictor of mortality. Survival curves were generated using Kaplan-Meier methods. Statistical analyses were conducted using SAS 9.4 (SAS Institute Inc.). Statistical significance was defined as a 2-tailed $P$ value $<.05$. Use of data in this project was authorized under Section 45 of Ontario's Personal Health Information Protection Act, which does not require review by a Research Ethics Board.

\section{Results}

A total of 9,441 patients were diagnosed with DLBCL in Ontario, Canada, between January 1, 2005, and December 31, 2015. Median age was 66 years, and 53.6\% were male. Median number of ADGs was 10 (interquartile range [IQR], 8-13), with $59.9 \%$ of patients having $\geq 10$ ADGs. Patients with treatment-naïve transformed FL accounted for $8.9 \%$ of the cohort (Table 1 ).

Median DTI was 37 days (IQR, 22-61 days), with $25.2 \%$ of patients waiting $>60$ days for treatment initiation (Table 2). Median time from diagnosis to hematology/oncology specialist review was 18 days (IQR, 8-32 days), followed by a median consult to treatment time of 15 days (IQR, 7-29 days). Median number of cycles of immunochemotherapy was 6 (IQR, 4-6 cycles), with $61.8 \%$ of patients completing $\geq 6$ cycles. A total of $64.1 \%$ of patients lived $<20 \mathrm{~km}$ from the treating center, with $12.9 \%$ traveling $\geq 60 \mathrm{~km}$. Between treatment initiation and study end on March 31, 2017, 43\% of patients died and a further $8 \%$ were censored for a new primary 


\begin{tabular}{|c|c|}
\hline Characteristic & n (\%) \\
\hline Total, N & 9,441 \\
\hline \multicolumn{2}{|l|}{ Age } \\
\hline Mean $\pm S D, y$ & $64.2 \pm 14.6$ \\
\hline Median (IQR), y & $66(55-75)$ \\
\hline Male sex & $5,062(53.6 \%)$ \\
\hline \multicolumn{2}{|l|}{ Income quintile } \\
\hline Missing & $35(0.4 \%)$ \\
\hline Q1 (lowest) & $1,566(16.6 \%)$ \\
\hline Q2 & $1,845(19.5 \%)$ \\
\hline Q3 & 1,841 (19.5\%) \\
\hline Q4 & 1,992 (21.1\%) \\
\hline Q5 (highest) & $2,162(22.9 \%)$ \\
\hline \multicolumn{2}{|l|}{ Cancer type } \\
\hline DLBCL & 8,601 (91.1\%) \\
\hline Transformed FL & 840 (8.9\%) \\
\hline \multicolumn{2}{|l|}{ Cancer stage $^{a}$} \\
\hline Missing & $5,119(54.2 \%)$ \\
\hline I & 965 (10.2\%) \\
\hline ॥ & $1,042(11.0 \%)$ \\
\hline III & 804 (8.5\%) \\
\hline IV & $1,510(16.0 \%)$ \\
\hline \multicolumn{2}{|l|}{ Comorbidities $^{b}$} \\
\hline$A D G s$, mean $\pm S D, n$ & $10.5(3.6)$ \\
\hline ADGs, median (IQR), $n$ & $10(8-13)$ \\
\hline ADG high $(\geq 10)$ & $5,650(59.9 \%)$ \\
\hline ADG $\bmod (6-9)$ & 2,987 (31.6\%) \\
\hline ADG low (0-5) & 804 (8.5\%) \\
\hline \multicolumn{2}{|l|}{ Rural dwelling } \\
\hline Urban & $8,170(86.5 \%)$ \\
\hline Rural & 1,271 (13.5\%) \\
\hline
\end{tabular}

Abbreviations: $A D G s$, aggregated diagnostic groups; $D L B C L$, diffuse large B-cell lymphoma; $F L$, follicular lymphoma; IQR, interquartile range; $\mathrm{Q}$, quintile. ${ }^{a}$ Cancer stage available from 2007 onwards.

${ }^{\mathrm{b}} \mathrm{ADG}$ s exclude cancer.

cancer (Figure 1). Median observation period in the rest of the cohort was 5.7 years from treatment initiation. Of the $43 \%$ of patients who died, lymphoma was listed as the primary cause in $73 \%$ of cases, with $9.3 \%(n=874)$ dying while on active treatment. Regardless of censoring, median time from diagnosis to death was 1.4 years (IQR, 0.6-4.7 years), and median time from treatment initiation to death was 1 year (IQR, $0.4-2.8$ years); $7 \%$ of patients died within 90 days of the first rituximab dose.

Among 8,565 patients (91\% of cohort) commencing therapy within 180 days of diagnosis date and who had

\section{Table 2. Clinical Characteristics}

\begin{tabular}{|lc|}
\hline Characteristic & $\mathbf{n}(\%)$ \\
\hline \multicolumn{1}{|l}{ Total, $\mathrm{N}$} & 9,441 \\
\hline $\begin{array}{l}\text { Number of completed chemotherapy cycles } \\
\text { Median (IQR), } \mathrm{n}\end{array}$ & $6(4-6)$ \\
\hline 6 & $4,057(43.0 \%)$ \\
\hline 7 & $343(3.6 \%)$ \\
\hline 8 & $1,435(15.2 \%)$ \\
\hline \begin{tabular}{l} 
Distance to first treatment facility \\
\hline Median (IQR), km
\end{tabular} & $11.4(5-31)$ \\
\hline$<20 \mathrm{~km}$ & $6,047(64.1 \%)$ \\
\hline $20-39.9 \mathrm{~km}$ & $1,441(15.3 \%)$ \\
\hline $40-59.9 \mathrm{~km}$ & $660(7.0 \%)$ \\
\hline$\geq 60 \mathrm{~km}$ & $1,221(12.9 \%)$ \\
\hline Unknown & $72(0.7 \%)$ \\
\hline Time from diagnosis to initiation of treatment & $37(22-61)$ \\
\hline Median (IQR), d & $3,496(37.0 \%)$ \\
\hline$<30 \mathrm{~d}$ & $3,570(37.8 \%)$ \\
\hline $30-60 \mathrm{~d}$ & $2,375(25.2 \%)$ \\
\hline$>60 \mathrm{~d}$ & $18(8-32)$ \\
\hline $\begin{array}{l}\text { Days from diagnosis to first consultation, } \\
\text { median (IQR) }\end{array}$ & $15(7-29)$ \\
\hline $\begin{array}{l}\text { Days from first consultation to treatment, } \\
\text { median (IQR) }\end{array}$ & \\
\hline
\end{tabular}

Abbreviation: IQR, interquartile range.

available data on distance to treatment facility, we observed a nonlinear association between DTI and OS; the nonlinear relationship between the log hazard of event and continuous DTI in days was U-shaped and is illustrated in Figure 2. This indicated that the cohort consisted of 3 patient groups: (1) those requiring immediate treatment, likely because of disease severity at presentation; (2) patients receiving treatment much later after diagnosis, potentially because of less-aggressive disease behavior; and (3) patients in the middle of the distribution. Kaplan-Meier OS curves of DTI, stratified by quintiles, are shown in Figure 3.

Adjusting for covariates, shorter DTI remained a significant predictor of mortality $(P<.001)$. In comparison with DTI of 0-18 days, those who commenced therapy at 19-29 days (HR, 0.75; 95\% CI, 0.68-0.84), 30-41 days (HR, 0.70; 95\% CI, 0.63-0.78), 42-57 days (HR, 0.52; 95\% CI, 0.46-0.58), and 58-180 days (HR, 0.52; 95\% CI, $0.47-0.58)$ had improved survival. Increasing age (HR, 1.03; 95\% CI, 1.03-1.04), male sex (HR, 1.23; 95\% CI, 1.14-1.32) and increasing number of ADG comorbidities (HR, 1.12; 95\% CI, 1.11-1.13) were associated with inferior survival (Table 3). We checked for collinearity between distance to treatment and DTI but observed no correlation, allowing us to test both exposure variables in 


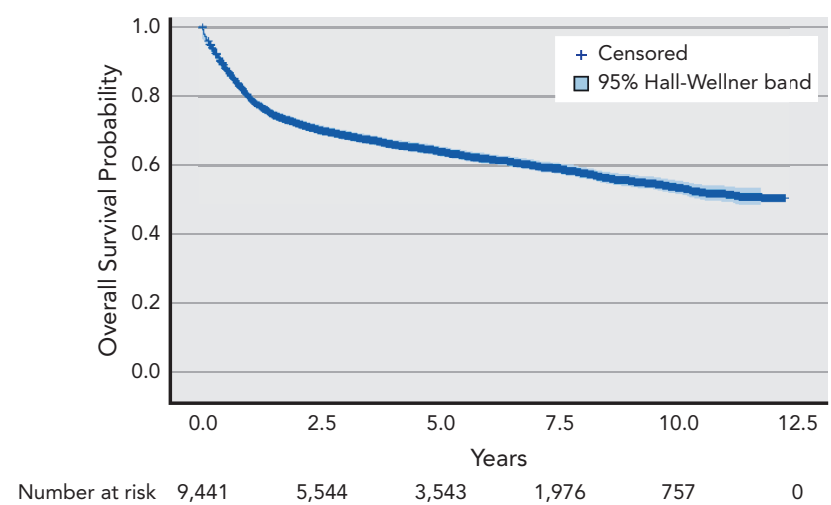

Figure 1. Overall survival for entire cohort $(n=9,441)$ from time of first treatment.

the model. We did not find an association between OS and distance traveled for treatment, and therefore removed it from the final model.

\section{Discussion}

We demonstrate a clinically significant impact of DTI in a population-based setting. Patients with shorter time between newly diagnosed DLBCL and treatment initiation have significantly poorer survival outcomes. We postulate that shorter DTI is a surrogate marker for more aggressive disease biology, resulting in the need for emergent therapy. This finding was independent of age, sex, comorbidities, and socioeconomic factors.

Our results complement the work published by Maurer et $\mathrm{al}^{6}$ who demonstrated that a longer DTI was associated with improved EFS at 24 months in 2 large prospective study populations, independent of the International Prognostic Index. In contrast, our study consists of an unselected general population of new DLBCL diagnoses. We also report the significant impact

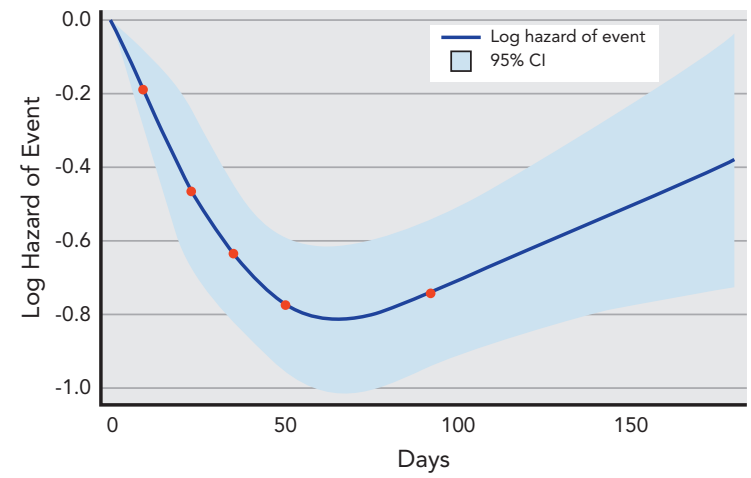

Figure 2. Effect of time between date of diagnosis and initiation of rituximab-based treatment on survival. Days are categorized using restricted cubic splines. Dots represent quintiles of diagnosis to treatment initiation.

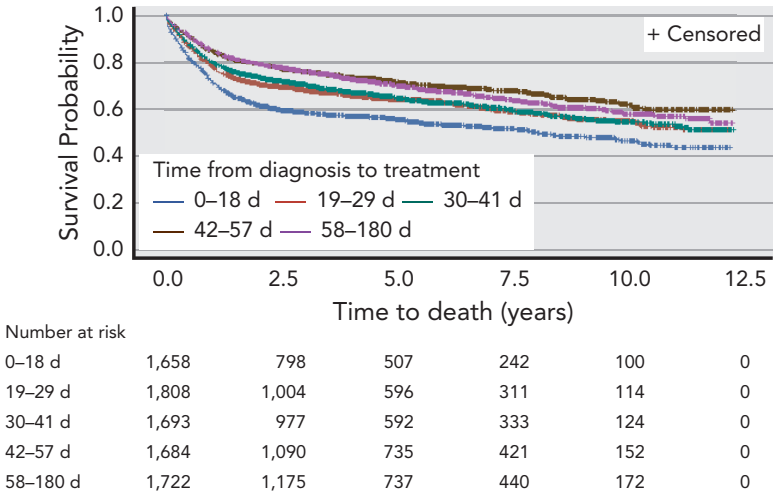

Figure 3. Kaplan-Meier curves of the probability of survival by diagnosis-to-treatment interval, stratified by quintiles.

that DTI had on OS, a finding supported by other population-based studies. An NCDB retrospective review demonstrated an improvement in 3-year OS estimate by $18 \%$ in patients with DLBCL treated $>30$ days from diagnosis compared with those treated within 0 to 7 days. ${ }^{7}$ A retrospective study of 689 patients with DLBCL reported inferior 5-year OS estimates in patients with earlier chemotherapy initiation $(<4$ weeks) versus initiation within 5 to 8 weeks from diagnosis $(61 \%$ vs $74 \%$, respectively; $P=.006) .{ }^{10}$ A smaller retrospective monocentric study of 345 patients reported similar findings. ${ }^{11}$

Frequency of emergency presentations across cancer types increases with age and is associated with a significant impact on 1-year survival. ${ }^{12}$ Although the observational design of our study prohibits the analysis of features of disease biology, our data support the observation that physicians expedite treatment initiation when aggressive features are present. In a populationbased study of 1,660 patients with DLBCL in the United Kingdom, emergency diagnoses were associated with more advanced stage, poorer physical fitness, decreased probability of treatment with curative intent, and poorer survival compared with similar patients diagnosed through alternative routes. ${ }^{13}$ Our group previously reported that patients treated within 1 week of diagnosis and those receiving the first cycle of chemotherapy as an inpatient had worse outcomes. ${ }^{14}$ Patients requiring emergent treatment are less likely to be considered for clinical trials, thus excluding those with the worst prognosis and creating a time-dependent selection bias. A time-dependent selection bias was previously proposed to explain the marked difference in DTI between a trial cohort and an academic referral group (23 vs 15 days). ${ }^{6}$ Furthermore, selection bias may explain the improved outcomes in control arms in recent trials.

To overcome the poorer prognosis in ABC DLBCL, cell of origin classification generated much enthusiasm 


\begin{tabular}{|c|c|}
\hline Variable & Adjusted $^{b}$ HR $(95 \% \mathrm{Cl})$ \\
\hline \multicolumn{2}{|l|}{ Time from diagnosis to treatment } \\
\hline $0-18 d$ & Ref \\
\hline $19-29 d$ & $0.75(0.68-0.84)$ \\
\hline $30-41 d$ & $0.70(0.63-0.78)$ \\
\hline $42-57 d$ & $0.52(0.46-0.58)$ \\
\hline $58-180 \mathrm{~d}$ & $0.52(0.47-0.58)$ \\
\hline Age (continuous) & $1.03(1.03-1.04)$ \\
\hline \multicolumn{2}{|l|}{ Sex } \\
\hline Female & Ref \\
\hline Male & $1.23(1.14-1.32)$ \\
\hline \multicolumn{2}{|l|}{ Neighborhood income quintile } \\
\hline Q1 (lowest) & Ref \\
\hline $\mathrm{Q} 2$ & $0.90(0.81-1.01)$ \\
\hline Q3 & $0.94(0.84-1.06)$ \\
\hline Q4 & $0.80(0.72-0.90)$ \\
\hline Q5 (highest) & $0.73(0.65-0.82)$ \\
\hline \multicolumn{2}{|l|}{ Rural dwelling } \\
\hline Rural & Ref \\
\hline Urban & $0.99(0.89-1.10)$ \\
\hline Number of ADG comorbidities (continuous) & $1.12(1.11-1.13)$ \\
\hline \multicolumn{2}{|l|}{ Lymphoma subtype } \\
\hline DLBCL & Ref \\
\hline Transformed FL & $0.92(0.79-1.07)$ \\
\hline \multicolumn{2}{|l|}{ Treatment facility type } \\
\hline Nonteaching & Ref \\
\hline Teaching & $0.93(0.86-1.00)$ \\
\hline
\end{tabular}

Abbreviations: ADG, aggregated diagnostic groups; $D L B C L$, diffuse large B-cell lymphoma; $F L$, follicular lymphoma; HR, hazard ratio; $Q$, quintile. alndividuals were followed from index (first rituximab treatment) until death, occurrence of a new primary cancer, or March 31, 2017.

${ }^{b}$ Adjusted for age, sex, distance to treatment facility, neighborhood income quintile, rural dwelling status, number of ADGs, cancer type, treatment facility type, and inpatient status at treatment.

for adding therapies targeting the NF-кB pathway. Thus far, the addition of novel agents to R-CHOP have had limited success. Adding bortezomib to R-CHOP (VR$\mathrm{CHOP}$ ) in a phase I/II trial showed early promise with similar progression-free survival (PFS) and OS in GCB and non-GCB cohorts. ${ }^{15}$ However, outcomes in a randomized phase II trial of VR-CHOP versus R-CHOP in non-GCB DLBCL were not significantly improved. ${ }^{5}$ The phase II REMODEL trial also reported negative findings when substituting bortezomib for vincristine in R-CHOP. ${ }^{16}$ Similarly, preclinical and early-phase data for lenalidomide $^{17,18}$ and ibrutinib ${ }^{19}$ added to R-CHOP were encouraging. The long-term results of 2 independent phase II studies suggested that R-CHOP + lenalidomide $\left(\mathrm{R}^{2}\right.$-CHOP) mitigated the negative prognosis associated with non-GCB DLBCL. ${ }^{18,20}$ However, in the phase III ROBUST trial, $\mathrm{R}^{2}$-CHOP failed to improve PFS or 2-year OS in patients with ABC DLBCL. ${ }^{3}$ It has been argued that differences in DTI (median DTI, 21 vs 31 days, respectively), higher-risk patient cohorts, and smaller population size between the phase II and III trials may account for these differences in outcome. ${ }^{21}$ In the PHOENIX trial, adding ibrutinib to R-CHOP also failed to demonstrate a benefit in non-GCB DLBCL. ${ }^{4}$ The placebo/R-CHOP arm had improved outcomes compared with historic studies, implying that factors other than cell of origin contributed to negative results. A subanalysis on patients aged $<60$ years showed a benefit in EFS, PFS, and OS compared with R-CHOP alone; however, further studies are needed to confirm these data.

The impact of social factors on outcomes remain uncertain. In a California population study of 33,032 patients with DLBCL from 1988 to 2009, those living in lower socioeconomic neighborhoods had a $34 \%$ higher all-cause mortality rate than those living in higher socioeconomic neighborhoods. ${ }^{22}$ The disparity was more prevalent among younger patients ( $<65$ years), those who were married, and after the introduction of rituximab, suggesting socioeconomic barriers to treatment. In our study, we demonstrate those in higher income quintiles had improved OS compared with their lowerincome counterparts. Other regions/countries have not reported such discrepancies. A United Kingdom study of 2,137 patients with DLBCL concluded that performance status rather than socioeconomic status was predictive of outcome, irrespective of age. ${ }^{23} \mathrm{~A}$ multicenter French study of 1,165 patients diagnosed between 2002 and $2008^{24}$ and a Dutch study of 343 consecutive diagnoses between 2005 and $2012^{25}$ both failed to identify disparities in outcome based on socioeconomic status. Similarly, a British Columbia Cancer Agency cohort showed no correlation between socioeconomic status and outcome. ${ }^{10}$

The impact of distance to treatment facility on outcomes also remains uncertain. We report no association between distance to treatment center and OS. However, a US-based population cohort consisting of 775,999 patients with newly diagnosed prostate cancer in 2004 through 2012 reported that traveling a longer distance was associated with a reduction in overall mortality rate. They postulated that this survival advantage was due to higher socioeconomic status, improved treatment availability, and academic, tertiary treatment facilities. ${ }^{26}$ An association between distance to treatment center and patient outcome was not replicated in a retrospective French cohort of 1,165 patients with 
DLBCL, which demonstrated that although patients treated in a teaching hospital had improved outcomes, those traveling $>15$ minutes to their treatment center had statistically worse outcomes. ${ }^{24}$

Limitations of our study include the observational nature of the design and lack of biologic data, such as DLBCL subtype based on cell of origin, stage, and prognostic indices such as the International Prognostic Index and lactate dehydrogenase level. Lymphomarelated endpoints, such as PFS, were also unavailable nor did we have certain patient-specific factors such as ECOG and frailty scores.

Our study represents, to our knowledge, the largest retrospective cohort of patients with newly diagnosed DLBCL treated within a publicly funded setting, which is a significant strength. Further strengths of our study include the use of multiple provincial-wide databases (with their inherent nature of comprehensive data collection), as well as the inclusion of comorbidities and sociodemographic characteristics as variables in the model.

\section{Conclusions}

A shorter DTI in patients with newly diagnosed DLBCL predicts for inferior OS in a real-world setting, likely reflecting a more-aggressive disease biology. Clinical trials with extended screening periods may be inadvertently enriched with patients with lymphoma that exhibits less-aggressive clinical behavior (and improved prognosis).

Our article adds to the growing evidence of a likely time-dependent selection bias for clinical trials caused by nonrecruitment of patients with shorter DTI.
Although many trials are reporting DTI, this is usually a key performance indicator, with the impact of DTI on overall response rarely reported. Trial designs should modify enrollment to capture patients who require emergency therapy and cannot undertake prolonged screening periods. This would help reduce timedependent patient selection bias and more accurately reflect the real-world experiences of patients with DLBCL.

\section{Acknowledgments}

Dr. Cheung receives funding from the Roy and Marjorie Linden Fund and the Joan Fisher and James Rowland Fund.

Submitted June 18, 2020; final revision received September 13, 2020 ; accepted for publication September 15, 2020.

Published online March 10, 2021.

Previous presentation: Parts of this study were presented as a poster at the 60th American Society of Hematology Annual Meeting and Exposition; December 1-4, 2018; San Diego, California. Poster 4850.

Author contributions: Study concept and methodology: All authors. Data curation, formal analysis, resources and software and data validation: Nagamuthu, Gatov, Croxford. Project administration: Blunt, Smyth, Nagamuthu, Gatov. Study supervision: Croxford, Mozessohn, Cheung. Writing_original draft: Blunt, Smyth. Writing-review \& editing: Nagamuthu, Gatov, Croxford, Mozessohn, Cheung.

Disclosures: The authors have disclosed that they have not received any financial consideration from any person or organization to support the preparation, analysis, results, or discussion of this article.

Funding: This study was supported by ICES, which is funded by an annual grant from the Ontario Ministry of Health and Long-Term Care (MOHLTC). Parts of this material are based on data and/or information compiled and provided by the Canadian Institute for Health Information (CIHI), Cancer Care Ontario (CCO), and Service Ontario.

Disclaimer: The analyses, conclusions, opinions, and statements expressed herein are those of the authors, and not necessarily those of $\mathrm{ClHI}, \mathrm{CCO}$, or Service Ontario; no endorsement is intended or should be inferred.

Correspondence: Danielle N. Blunt, BMBS, Department of Haematology, Royal Adelaide Hospital, 1 Port Road, Adelaide, South Australia, Australia 5000 Email: danielle.blunt@sa.gov.au

\section{References}

1. Rosenwald A, Wright G, Chan WC, et al. The use of molecular profiling to predict survival after chemotherapy for diffuse large-B-cell lymphoma. N Engl J Med 2002;346:1937-1947.

2. Davis RE, Brown KD, Siebenlist U, et al. Constitutive nuclear factor kappaB activity is required for survival of activated B cell-like diffuse large B cell lymphoma cells. J Exp Med 2001;194:1861-1874.

3. Vitolo U, Witzig TE, Gascoyne RD, et al. ROBUST: first report of phase III randomized study of lenalidomide/RCHOP (R2-CHOP) vs placebo/R$\mathrm{CHOP}$ in previously untreated $\mathrm{ABC}$-type diffuse large B-cell lymphoma. Hematol Oncol 2019;37(Suppl 2):36-37.

4. Younes A, Sehn LH, Johnson P, et al. Randomized phase III trial of ibrutinib and rituximab plus cyclophosphamide, doxorubicin, vincristine, and prednisone in non-germinal center B-cell diffuse large B-cell lymphoma. J Clin Oncol 2019;37:1285-1295.

5. Leonard JP, Kolibaba KS, Reeves JA, et al. Randomized phase II study of $\mathrm{R}-\mathrm{CHOP}$ with or without bortezomib in previously untreated patients with non-germinal center B-cell-like diffuse large B-cell lymphoma. J Clin Oncol 2017;35:3538-3546.

6. Maurer MJ, Ghesquières H, Link BK, et al. Diagnosis-to-treatment interval is an important clinical factor in newly diagnosed diffuse large B-cell lymphoma and has implication for bias in clinical trials. J Clin Oncol 2018;36:1603-1610.

7. Olszewski AJ, Ollila T, Reagan JL. Time to treatment is an independent prognostic factor in aggressive non-Hodgkin lymphomas. Br J Haematol 2018;181:495-504.
8. Clarke EA, Marrett LD, Kreiger N. Cancer registration in Ontario: a computer approach. IARC Sci Publ 1991;95:246-257.

9. Harrell FE. Regression Modeling Strategies: With Applications to Linear Models, Logistic Regression, and Survival Analysis. New York, NY: Springer-Verlag; 2010.

10. Hay K, Lee B, Goktepe O, et al. Impact of time from diagnosis to initiation of curative chemotherapy on survival of patients with diffuse large B-cell lymphoma. Leuk Lymphoma 2016;57:276-282.

11. Camus V, Dubois S, Jardin F, et al. Prognostic impact of diagnosis to treatment interval (DTI) in diffuse large B-cell lymphoma patients: a reallife monocentric study. Leuk Lymphoma 2019;60:839-841.

12. Elliss-Brookes L, McPhail S, Ives A, et al. Routes to diagnosis for cancer-determining the patient journey using multiple routine data sets. $\mathrm{Br} \mathrm{J}$ Cancer 2012;107:1220-1226.

13. Kane E, Howell D, Smith A, et al. Emergency admission and survival from aggressive non-Hodgkin lymphoma: a report from the UK's populationbased Haematological Malignancy Research Network. Eur J Cancer 2017. 78:53-60.

14. Nikonova A, Guirguis HR, Buckstein R, et al. Predictors of delay in diagnosis and treatment in diffuse large B-cell lymphoma and impact on survival. Br J Haematol 2015;168:492-500.

15. Ruan J, Martin P, Furman RR, et al. Bortezomib plus CHOP-rituximab for previously untreated diffuse large B-cell lymphoma and mantle cell lymphoma. J Clin Oncol 2011:29:690-697. 
16. Offner F, Samoilova O, Osmanov E, et al. Frontline rituximab, cyclophosphamide, doxorubicin, and prednisone with bortezomib (VR-CAP) or vincristine (R-CHOP) for non-GCB DLBCL. Blood 2015;126:1893-1901.

17. Hernandez-llizaliturri FJ, Deeb G, Zinzani PL, et al. Higher response to lenalidomide in relapsed/refractory diffuse large B-cell lymphoma in nongerminal center B-cell-like than in germinal center B-cell-like phenotype. Cancer 2011;117:5058-5066.

18. Nowakowski GS, LaPlant B, Macon WR, et al. Lenalidomide combined with R-CHOP overcomes negative prognostic impact of non-germinal center B-cell phenotype in newly diagnosed diffuse large B-cell lymphoma: a phase II study. J Clin Oncol 2015;33:251-257.

19. Wilson $\mathrm{WH}$, Young RM, Schmitz $\mathrm{R}$, et al. Targeting $B$ cell receptor signaling with ibrutinib in diffuse large B cell lymphoma. Nat Med 2015;21:922-926.

20. Castellino A, Chiappella A, LaPlant BR, et al. Lenalidomide plus $\mathrm{R}-\mathrm{CHOP} 21$ in newly diagnosed diffuse large B-cell lymphoma (DLBCL): long-term follow-up results from a combined analysis from two phase 2 trials. Blood Cancer J 2018;8:108

21. Nowakowski G, Chiappella A, Hong F, et al. Potential factors that impact lenalidomide/R-CHOP efficacy in previously untreated diffuse large B-cell lymphoma in the ROBUST and ECOG-ACRIN 1412 studies [abstract]. Blood 2019;134(Suppl 1):4092. Abstract 626.

22. Tao L, Foran JM, Clarke CA, et al. Socioeconomic disparities in mortality after diffuse large B-cell lymphoma in the modern treatment era. Blood 2014; $123: 3553-3562$

23. Smith A, Crouch S, Howell D, et al. Impact of age and socioeconomic status on treatment and survival from aggressive lymphoma: a UK population-based study of diffuse large B-cell lymphoma. Cancer Epidemiol 2015:39:1103-1112.

24. Le Guyader-Peyrou S, Orazio S, Dejardin O, et al. Factors related to the relative survival of patients with diffuse large B-cell lymphoma in a population-based study in France: does socio-economic status have a role? Haematologica 2017;102:584-592.

25. Boslooper K, Hoogendoorn M, van Roon EN, et al. No outcome disparities in patients with diffuse large B-cell lymphoma and a low socioeconomic status. Cancer Epidemiol 2017;48:110-116.

26. Vetterlein MW, Löppenberg B, Karabon P, et al. Impact of travel distance to the treatment facility on overall mortality in US patients with prostate cancer. Cancer 2017:123:3241-3252.

\section{RECORDED PRESENTATIONS}

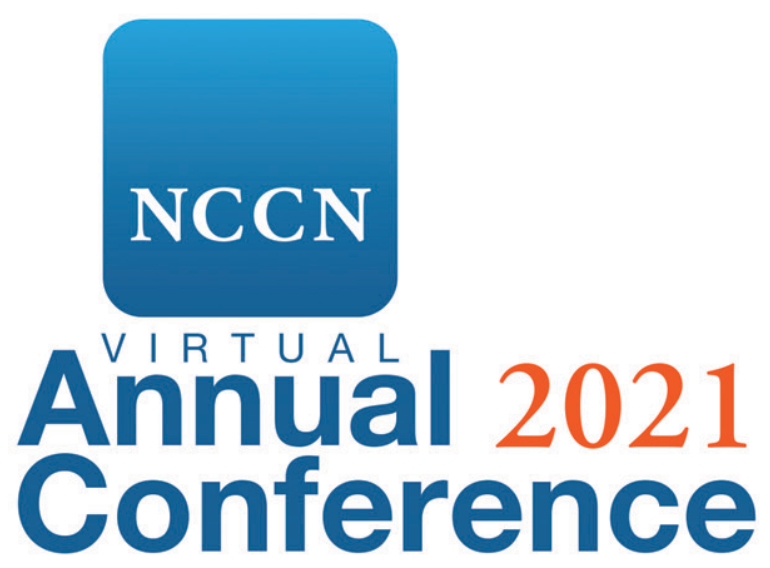

education.nccn.org/ac2021

\section{Featuring over 35 educational sessions and challenging case reviews!}

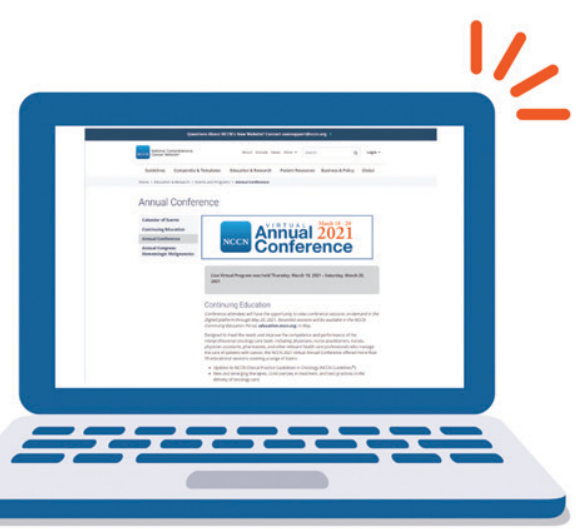

